\title{
Association of SCN1A Mutations with Epilepsy among Sudanese Patients
}

\author{
Sanaa Abdalaziz Mohamed ${ }^{*}$, Sawsan A.H. Aldeaf², Rasha Elhassan², Abasshar Hussein ${ }^{4}$, \\ Alsadig Gassoum ${ }^{2,3}$, Abd Elkarim A. Abdrabo1 (1) \\ ${ }^{1}$ Department of Clinical Chemistry, Faculty of Medical Laboratory Sciences, Al-Neelain University, Khartoum, Sudan \\ ${ }^{2}$ National Center of Neurological Science, Khartoum, Sudan \\ ${ }^{3}$ Almadain College for Medical Science and Technology, Khartoum, Sudan \\ ${ }^{4}$ Department of Medicine, Faculty of Medicine, University of Khartoum, Khartoum, Sudan \\ Email: *abdrabokarim@gmail.com
}

How to cite this paper: Mohamed, S.A., Aldeaf, S.A.H., Elhassan, R., Hussein, A., Gassoum, A. and Abdrabo, A.E.A. (2021) Association of SCN1A Mutations with Epilepsy among Sudanese Patients. Neuroscience \& Medicine, 12, 90-102.

https://doi.org/10.4236/nm.2021.123008

Received: May 2, 2021

Accepted: September 25, 2021

Published: September 28, 2021

Copyright (c) 2021 by author(s) and Scientific Research Publishing Inc. This work is licensed under the Creative Commons Attribution International License (CC BY 4.0).

http://creativecommons.org/licenses/by/4.0/

\begin{abstract}
Background: Genetics research of humans has established that a genetic basis contributes to the susceptibility to epilepsy for a majority of the cases. Although many epilepsies are secondary to injury or another illness, approximately $40 \%$ are idiopathic, meaning that the original cause is unknown. It is presumed that most idiopathic epilepsies result from genetic abnormalities, with the majority likely caused by mutations in multiple currently unidentified genes. However, research has revealed a growing number of single-gene mutations that cause epilepsy. Objective: To detect some of the genetic mutations which may cause idiopathic epilepsy. Methods: The current study is a cross-sectional study that had been performed at Sheikh Mohamed Khair center, Banat, Omdurman, and National Centre for Neurological Sciences (NCNS) Khartoum state, during the period 2016 to 2019. Ninety-nine participants were enrolled in this study. Demographic data were collected in a predesigned questionnaire blood samples were analyzed for biochemical and molecular tests. Results: Ninety-nine patients diagnosed with idiopathic epilepsy were recruited in this study. The most affected age group was $18-40$ years accounted for $55 \%$ of patients. Females were the majority with $53 \%$. Fifty percent of the patients had the first seizure at age less than 5 years. Ninety percent of the patients have no Family history with epilepsy. All sequenced samples showed genetic mutations, deletion mutation was detected in $71 \%$ of the samples. Bioinformatics tools detected a frameshift mutation in the chain of the amino acids. Conclusion: The current study detected deletion mutations in SCN1A gene (frameshift) can cause epilepsy by changing some amino acids with residues that can affect neuronal stability indirectly.
\end{abstract}




\section{Keywords}

Epilepsy, National Centre for Neurological Science, Sudan, SCN1A

\section{Introduction}

Epilepsy is one of the most frequent neurological disorders; the prevalence estimation for active epilepsy ranges from $0.2 \%$ to $4.1 \%$ [1]. It is a pathological condition characterized by repeated, unprovoked, epileptic seizures [2]. Diverse causes of epilepsy are commonly being recognized, such as structural, metabolic, or genetic causes; yet, the majority of epilepsies have unknown etiology [3]. It is not surprising that alterations in the ion channels may be a pathogenetic cause, and indeed, at least for some syndromes, epilepsies are increasing thought to be "channelopathies" either due to genetic mutations or as the endpoint of hitherto unknown pathological processes [4]. The voltage-gated $\mathrm{Na}^{+}$channels' primary role is the initiation of action potentials, making them critical determinants of neuronal excitability [5]; it consists of large a subunits which connect with further polypeptides, for instance, $\beta$ subunits to outline efficient voltage-gated ion channels [6]. Defects in sodium channels subunits make them susceptible to slow inactivation, i.e., the membrane stays depolarized for a longer time, as a result, it can cause epileptogenesis and increase of seizures [7] [8]. It should be mentioned that changes in sodium channels had been detected in brain tissues of epileptic patients, suggestive of a potential role for sodium channels in the pathophysiology of epilepsy [9].

SCN1A encodes the NaV1.1 subunit expressed mostly in inhibitory GABAergic neurons and is enriched at the axon initial segment, implicating a role in the beginning and the spread of action potentials in these cells [10] [11]. It was first implicated in epilepsy in 2000 [12]. Several hundred mutations of SCN1A have been reported in epilepsy since first discovered, making it the most frequently known epilepsy gene [13].

This study aims to detect some of the possible causes of idiopathic epilepsy by analyzing a sequence of SCN1A gene in addition to calcium levels.

\section{Materials and Methods}

This study is a cross-sectional study that was performed at Sheikh Mohamed Khair centre, Khartoum state. This center is a primary health care center however idiopathic epilepsy diagnosis was provided by neurology physician depending essentially on the description of eyewitness to the seizure attack. Ninety-nine participants were enrolled in this study starting November 2016 to February 2019. Only Patients who were not diagnosed with idiopathic epilepsy and those who refused to patients were excluded from this study. Patients' demographic, and clinical data including age, gender, ethnicity, class of seizure, the onset of 
seizure, frequency of seizure, seizure classification, precipitating factor, modality of treatment psychosocial aspects, concomitant illness, and family history were recorded in a predesigned questionnaire. Analyses were performed using Microsoft Office Excel 2010, and Statistical Package for Social Science Program (SPSS version 25). Blood samples were collected from each patient in two containers, EDTA container and LI-heparin, the EDTA samples were processed for DNA extraction, and the heparinized samples were used for calcium estimation.

\section{Biochemical Analysis}

Plasma was separated by centrifuge (Hettich Zenterfuge EBA200, Kirchlengern, Germany) at 3000 RPM for 5 minutes and then stored at $-20^{\circ} \mathrm{C}$. Calcium concentrations were obtained by semi-automated chemistry Analyzer (Mindary BA-88A).

\section{Molecular Genetic Analysis}

Extraction of deoxyribonucleic acid (DNA) was performed from whole blood samples using QIAGEN ${ }^{\circledR}$ DNA extraction kits (vacuum protocol).

SCN1A gene was amplified using polymerase chain reaction (PCR), in PCR tube $20 \mu \mathrm{L}$ of readymade Master Mix $\left(4 \mu \mathrm{L}\right.$ of $5 \times$ Firepol $^{\circledR}$ Master Mix (Solis Bio-Dyne, Tartu, Estonia) was added to $1 \mu \mathrm{L}$ forward primer, $1 \mu \mathrm{L}$ reverse primer, and $14 \mu \mathrm{L}$ distilled water), followed by $2 \mu \mathrm{L}$ of DNA.

Primers used for amplification were 5-TACCCTGTTCCGAGTGATCC3 forward primer and 5'-GCTGTTGCCAAAGGTCTCAA3' reverse primer, then the amplified PCR products were separated using $2 \%$ gel electrophoresis. Then, separated DNA was visualized using UV light.

\section{DNA Sequencing}

Fifteen PCR products were sent to China for sequencing (BGI solutions co. LTD).

\section{Data Analysis}

\section{Sequencing Analysis}

The sequencing results were analyzed using multiple bioinformatics software and tools. The nucleotides sequences of SCN1A gene were searched for sequences similarity using nucleotide BLAST NCBI (https://www.ncbi.nlm.nih.gov/) then subjected to multiple sequence alignment using Bio-Edit software. The detected mutations were analyzed using bioinformatics tools, the information regarding the detected SNPs were obtained from National Center for Biological Information (NCBI), as for predicting damaging amino acid substitutions, mutation taster was used in addition to HOPE.

\section{Statistical Analysis}

Demographic, clinical, and sequencing findings were performed by Microsoft Office Excel, and Statistical Package for Social Science Program 2010 SPSS version 25 , which included frequencies, cross-tabulation. 


\section{Result}

\subsection{Demographic Results}

Ninety-nine patients diagnosed with idiopathic epilepsy were recruited in this study, Highest age group category was 18 - 40 years accounted for $55 \%$ of Patients, followed by less than 18 years group (32\%), then 41 - 65 years (11\%) while more than 65 years group were only $2 \%$ (Table 1 ).

Regarding gender, females were the majority with $53 \%$ and $47 \%$ males (Table 2).

Fifty percent of the patients had the first seizure at age less than $5 \mathrm{yrs}, 28 \%$ of them had it in age between $5-10$ years $22 \%$ had the onset of seizure at age more than 10 years (Table 3 ).

Ninety percent of the patients have no Family history with epilepsy while the rest of them (10\%) declared to have a family history (Table 4 ).

\subsection{Biochemical Results}

Cross-tabulation of calcium among patients revealed calcium level $<8.5 \mathrm{mg} / \mathrm{dL}$ was found in $54.4 \%$ of the patients followed by $39.4 \%$ with normal calcium level (8.5 - 10.5) (Table 5).

Table 1. The age groups in idiopathic epileptic patients.

\begin{tabular}{cccc}
\hline Age & No of patients & $\%$ & P-value \\
\hline More than 65 years & 2 & $2.0 \%$ & $<0.001$ \\
Less than 18 years & 32 & $32.0 \%$ & \\
$18-40$ years & 55 & $55.0 \%$ & \\
\hline
\end{tabular}

Table 2. The gender domination in idiopathic epileptic patients.

\begin{tabular}{cccc}
\hline Gender & No of patients & $\%$ & P-value \\
\hline Female & 53 & $53.0 \%$ & 0.549 \\
Male & 47 & $47.0 \%$ & \\
\hline
\end{tabular}

Table 3. The onset of seizure in age groups among idiopathic epileptic patients.

\begin{tabular}{cccc}
\hline Onset of seizure & No of patients & $\%$ & P-value \\
\hline Less than 5 years & 50 & $50.0 \%$ & \\
More than 10 years & 22 & $22.0 \%$ & \\
$5-10$ years & 28 & $28.0 \%$ & \\
\hline
\end{tabular}

Table 4. The family history among idiopathic epileptic patients.

\begin{tabular}{cccc}
\hline Family history & No of patients & $\%$ & P-value \\
\hline No & 90 & $90.0 \%$ & $<0.001$ \\
Yes & 10 & $10.0 \%$ & \\
\hline
\end{tabular}




\subsection{Molecular and Sequencing Results}

In the current study, the sequencing findings of the SCN1A gene showed that, at chr2:166848853 $\mathrm{C}>\mathrm{G}$ was detected in (57\%) of the samples, while $\mathrm{C}>\mathrm{T}$ was detected in $14 \%$, both mutations showed splice site effect. Deletion AT was detected at chr2:166848848-49 in 71\% of samples. Also $\mathrm{C}>\mathrm{T}$ on chr2:166848847 was detected $(71 \%)$ with splice site effect, $\mathrm{T}>\mathrm{A}$ single base exchange was detected in (14\%) on chr2:166848841 with splice site effect, in addition, Deletion A at positions chr2:166848832 and chr2:166848824, were observed in $71 \%$ of the samples which appear to have a splice site effect (Figure 1).

\subsection{Bioinformatics Results}

Furthermore, SCN1A gene mutations were analyzed using mutation taster program (http://www.mutationtaster.org/) to obtain the type and location of mutations, while Hope database (https://www3.cmbi.umcn.nl/hope/) was used to detect the mutation impact upon the protein. The findings of mutation taster program showed that deletion AT and A created frame shift mutations with amino acid change at positions I1646P and G1652E respectively, Figures 2-5. In addition to that, deletion A showed amino acid change at position 1654 from Lysine to Arginine (Figure 6, Figure 7).

\section{Discussion}

Epilepsy is one of the most frequent neurological disorders; the pooled incidence rate of epilepsy was 61.4 per 100,000 person-years (95\% CI 50.7 - 74.4) [14] with a $3 \%$ risk of developing epilepsy across all ages [15].

Table 5. Calcium levels in idiopathic epileptic patients.

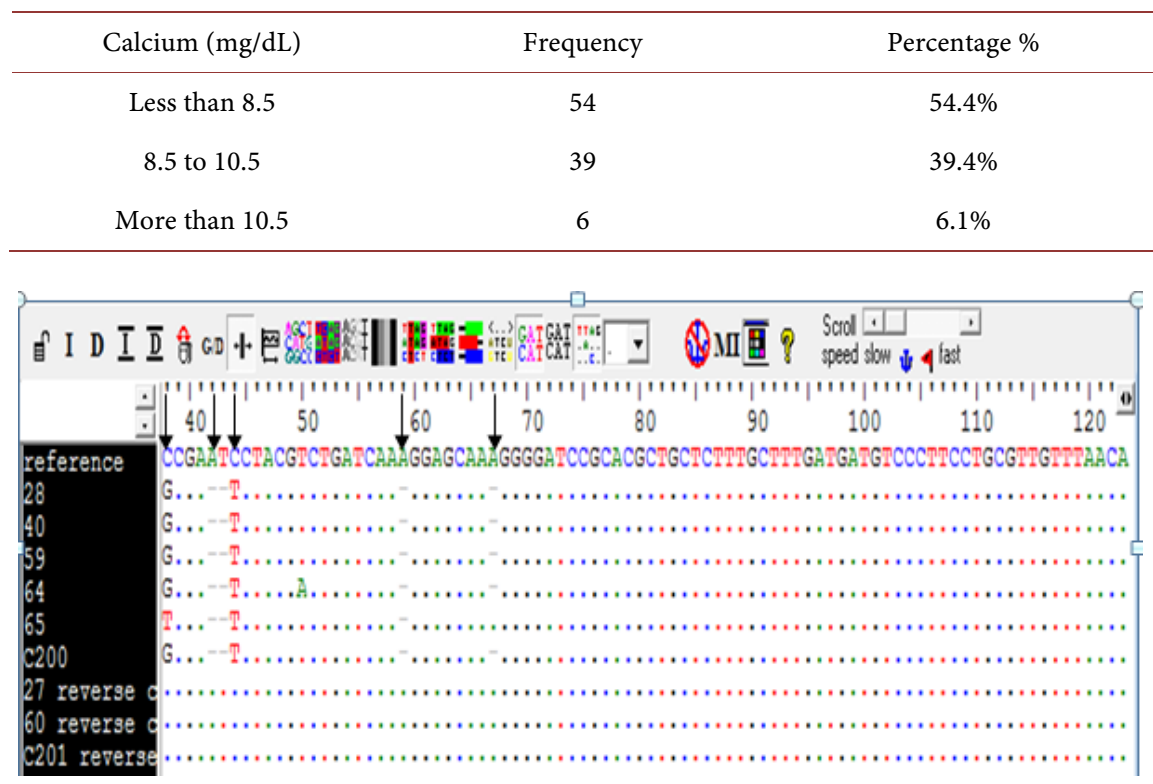

Figure 1. The alignment of SCN1A gene sequence obtained from NCBI database with our samples, using BioEdit program. Arrows indicates mutations sites. 
Mutatior

(⿻)

\section{Prediction disease causing}

Summary

- amino acid sequence changed

- frameshift

- protein features (might be) affected

- splice site changes

- truncated protein (might cause NMD)

analysed issue analysis result name of no title

alteration

alteration (phys. chr2:166848848_166848849delAT

location)

HGNC symbol SCN1A

Ensembl ENST00000423058

transcript ID

Genbank transoript

UniProt peptide P35498

alteration type deletion

alteration region CDS

DNA changes c.4936 4937delAT

CDNA.4954_4955delAT

g.81301_81302delAT

AA changes $11646 \mathrm{Pfs}^{\star} 26$

position(S) of 1646 (frameshift or PTC - further changes downstream)

altered AA

if $A A$ alteration in

CDS

frameshift yes

known variant Variant was neither found in EXAC nor $1000 \mathrm{G}$

Mutatio

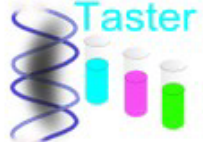

\section{mutation t@sting}

\section{Prediction disease causing}

Model: complex_aae, prob: 1 (explain

Summary

- amino acid sequence changed

- frameshift

protein features (might be) affected

- splice site changes

- truncated protein (might cause NMD)

analysed issue analysis result

name of notitle

alteration

no title

alteration (phys. chr2:166848824_166848824delT

Iocation)

HGNC symbol SCN1A

Ensembl ENST00000423058

transcript

Genbank transoript:

UniProt peptide P35498

alteration type deletion

alteration region CDS

DNA changes C.4961_4961delA

CDNA.4979 4979delA

g.81326_81326delA

AA changes K1654Rfs*10

position(S) of 1654 (frameshift or PTC - further changes downstream)

altered AA

if $A A$ alteration in

$\operatorname{CDS}$

frameshift yes

known variant Variant was neither found in ExAC nor $1000 \mathrm{G}$

Figure 2. Deletion AT mutation at chr2:166848848-166848849 using Mutation taster program. 


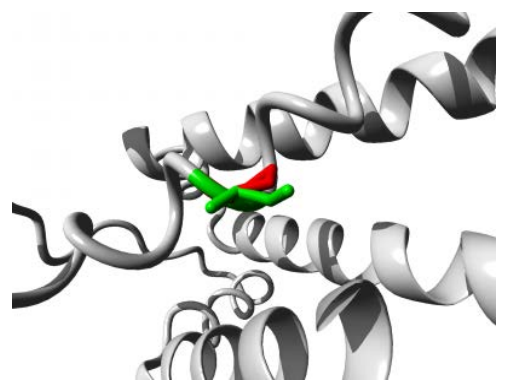

Figure 3. The wild and mutant aa residues at position (I1646P) using Hope program (PDB:6AGF). The wild type is in green and the mutant in red color.

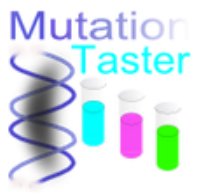

Prediction disease causing

Summary

- amino acid sequence changed

- frameshift

- protein features (might be) affected

- splice site changes

- truncated protein (might cause NMD)

analysed issue analysis result

name of no title

alteration

alteration (phys, chr2:166848832_166848832delT

location)

HGNC symbol SCN1A

Ensembl

transcript ID

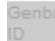

UniProt peptide P35498

alteration type deletion

alteration region CDS

DNA changes c.4953 4953delA

c. 4953 -4953delA

CDNA.4971 81318 - 81318 delA

AA changes G1652Efs $* 12$

position(s) of 1652 (frameshift or PTC - further changes downstream)

altered $\mathrm{AA}$

if $A A$ alteration in

CDS

frameshift

known variant Variant was neither found in ExAC nor 1000G.

Figure 4. Deletion A mutation at chr2:166848832-166848832 using Mutation taster program.

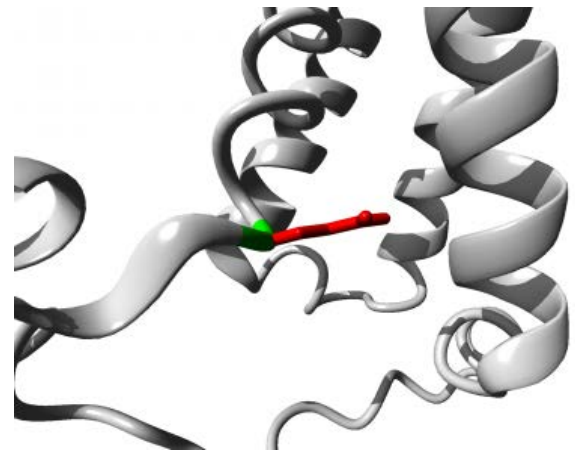

Figure 5. The wild and mutant aa residues at position (G1652E) using Hope program (PDB:6AGF). The wild type is in green and the mutant in red color. 


\section{Mutatior \\ iii}

Prediction disease causing

Summary

- amino acid sequence changed

- frameshift

- protein features (might be) affected

- splice site changes

- truncated protein (might cause NMD)

analysed issue

name of

alteration

alteration (phys. chr2:166848824_166848824delT

location)

HGNC symbol SCN1A

Ensembl ENST00000423058

transcript ID

Genbank transoript

UniProt peptide P35498

alteration type deletion

alteration region $\mathrm{CDS}$

DNA changes c.4961 4961delA

cDNA.4979_4979delA

g.81326_81326delA

AA changes

K1654Rfs*10

position(s) of

altered $A A$

if AA alteration in

$\operatorname{CDS}$

frameshift yes

1654 (frameshift or PTC - further changes downstream)

Figure 6. Deletion A mutation at chr2:166848824-166848824 using Mutation taster program.

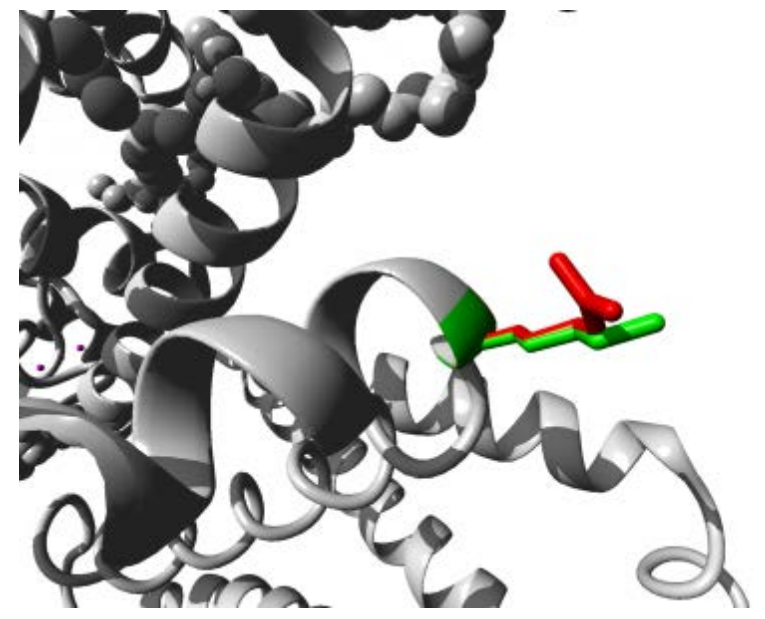

Figure 7. The wild and mutant aa residues at position (K1654R) using Hope program (PDB:6AGF). The wild type is in green and the mutant in red color.

The current study was performed on 99 patients with idiopathic epilepsy; it showed that $50 \%$ of the participants had onset of the disease in an age less than 5 years old. Though according to Marini Cet [16] there is a shred of increasing 
evidence for the existence of IGE beginning beyond the third decade.

Regarding gender, males were less than females 53\% females, $47 \%$ male. Similar findings were conducted in Asadi study [17] females 57\%, 43\% were males. Outnumbering of females over males is expected as Studies showed that female sex steroid hormones have repeatedly been shown to affect neuronal excitability [18].

The diagnosis of idiopathic epilepsy starts with a questionnaire establishing the Family History of Epilepsy (FHE) on these patients [19]. In this study, 90\% of the patients have no Family history with epilepsy while the rest of them (10\%) declared to have a family history. Unlike Babtain FA study results [20] which showed a significant association between idiopathic epilepsy and the presence of FHE.

Calcium level was normal (mean $8.32 \mathrm{mg} / \mathrm{dl}$ and SD 1.25), while A prospective case-control study done by Oladipo $\mathrm{O}$ [21] showed that calcium levels are lower in children with epilepsy compared to the controls during the seizure-free periods.

Nearly $70 \%$ of epileptic patients lack an obvious pathogenetic cause and genetics are believed to play a vital role in its causation [22] Voltage-gated sodium channels are an important group of ion channels, which play an important role in generating action potential and depolarization of the neurons. Mutation in the SCN1A gene causes defective gating in the sodium channel and thus causes hyperexcitability and seizures [23]. More recent studies have indicated that common variants in the SCN1A gene may be risk factors for common epilepsies like temporal lobe epilepsy and idiopathic/genetic generalized epilepsy (GGE/IGE) [24]. Factors that do not necessarily change the structure of the gene however do affect the progression of transcription and translation is recognized as epigenetic factors [25]. Any perturbation in the transcription, translation, or epigenetic mechanisms can generate defective proteins which lead to diseases. Seizures occur as a result of a complex interplay of altered gene expressions, increased neuronal excitability, and disturbed intrinsic neuronal properties [26] [27]. Defects in epilepsy genes give a critical insight into the pathomechanisms of seizure generation and propagation, which has an impact on the management of the patients [28].

In this study $\mathrm{C}>\mathrm{T}$ on chr2:166848847 mutation was detected with single base exchange alteration type and splice site effect, similar mutation was reported earlier in NCBI (rs777853016).

Also several deletions were detected on SCN1A gene which is located in chromosome 2, it was found that AT deletion at chr2: 166848848_166848849 changed the amino acid sequence at position 1646, protein features (might be) affected and the splice site had changed. As a result, isoleucine muted into a proline, with the size difference, mutation caused an empty space in the core of the protein. L proline is a GABA-analogue, its accumulation in the cytosol of GABAergic neurons causes competitive inhibition of Glutamic Acid Decarboxylase (GAD) leading to deficient gamma-aminobutyric acid (GABA) production [29], 
GABA is the main inhibitory neurotransmitter in the cerebral cortex, it maintains the inhibitory tone that counterbalances neuronal excitation. When this balance is disturbed, seizures may arise [30].

A deletion at chr2: 166848832_166848832 (frame-shift mutation) made a change in the amino acid sequence with glycine mutation into a glutamic Acid at position 1652. The most flexible of all residues is glycine. This flexibility might be required for the protein's function. Mutation of this glycine can eliminate this function.

The wild-type residue charge was neutral, while the mutant residue charge is negative, this can cause repulsion between the mutant residue and neighboring residues. Also, glycine location is on the surface of the protein, alteration of this residue can disturb interactions with other molecules or other parts of the protein. It should be mentioned that the torsion angles for glycine are unusual .only glycine is flexible enough to make these torsion angles, so any mutation will develop an incorrect confirmation which will disturb the local structure [31]. glutamic acid is considered to be a major excitatory neurotransmitter in the vertebrate CNS [32]. Studies conducted in the past few decades are evidence for the role of glutamic acid in epilepsy [33].

A >- was identified at 166848824_166848824, with a frame-shift mutation it caused a change in the amino acid sequence with mutation of a Lysine into an arginine at position 1654. Arginine, also known as 1 -arginine (symbol Arg or R), is an $\alpha$-amino acid that is used in the biosynthesis of proteins [34]. It is a precursor for the synthesis of Nitric Oxide (NO) [35]. Nitric Oxide (NO) is a shortlived, gaseous signaling molecule that is produced endogenously by a family of enzymes called the Nitric Oxide Synthases (NOS), which catalyze the synthesis of NO from the amino acid arginine [36] it have been pointed out as potential neurotransmitters or retrograde messengers [37] linked to synaptic plasticity [38] and regulation of brain excitability, including the triggering of seizure activity [39] [40]. The involvement of NO in epileptic disorders has been shown in experiments with systemic injection of NOS inhibitors [39] [41].

The study was limited by two factors, first, the sequencing analysis was done to selected samples, this is due to the limited financial source, this research was self funded and no any other institutional or governmental funded given. Secondly, the sample size is not like other published articles in the same field, that is because the majority of the patients refused to be included in the study and we didn't find enough collaboration with the co-patients and the patients.

\section{Conclusion}

A deletion mutation in SCN1A gene (frameshift) can cause epilepsy by changing some amino acids with residues that can affect neuronal stability indirectly.

\section{Conflicts of Interest}

The authors declare no conflicts of interest regarding the publication of this paper. 


\section{References}

[1] Ottman, R., Lipton, R.B., Ettinger, A.B., Cramer, J.A., Reed, M.L., Morrison, A., et al. (2011) Comorbidities of Epilepsy: Results from the Epilepsy Comorbidities and Health (EPIC) Survey. Epilepsia, 52, 308-315. https://doi.org/10.1111/j.1528-1167.2010.02927.x

[2] LaFrance, J.W.C., Baker, G.A., Duncan, R., Goldstein, L.H. and Reuber, M. (2013) Minimum Requirements for the Diagnosis of Psychogenic Nonepileptic Seizures: A Staged Approach: A Report from the International League against Epilepsy Nonepileptic Seizures Task Force. Epilepsia, 54, 2005-2018.

https://doi.org/10.1111/epi.12356

[3] Irani, S.R., Bien, C.G. and Lang, B. (2011) Autoimmune Epilepsies. Current Opinion in Neurology, 24, 146-153. https://doi.org/10.1097/WCO.0b013e3283446f05

[4] Celesia, G.G. (2001) Disorders of Membrane Channels or Channelopathies. Clinical Neurophysiology, 112, 2-18. https://doi.org/10.1016/S1388-2457(00)00496-X

[5] Alexander, S.P., Fabbro, D., Kelly, E., Marrion, N., Peters, J.A., Benson, H.E., et al. (2015) The Concise Guide to PHARMACOLOGY 2015/16: Enzymes. British Journal of Pharmacology, 172, 6024-6109. https://doi.org/10.1111/bph.13354

[6] Marban, E., Yamagishi, T. and Tomaselli, G.F. (1998) Structure and Function of Voltage-Gated Sodium Channels. The Journal of Physiology, 508, 647-657. https://doi.org/10.1111/j.1469-7793.1998.647bp.x

[7] Alekov, A.K., Rahman, M.M., Mitrovic, N., LehmannHorn, F. and Lerche, H. (2000) A Sodium Channel Mutation Causing Epilepsy in Man Exhibits Subtle Defects in Fast Inactivation and Activation In Vitro. The Journal of Physiology, 529, 533-540. https://doi.org/10.1111/j.1469-7793.2000.00533.x

[8] Vilin, Y.Y. and Ruben, P.C. (2001) Slow Inactivation in Voltage-Gated Sodium Channels. Cell Biochemistry and Biophysics, 35, 171-190.

https://doi.org/10.1385/CBB:35:2:171

[9] Mantegazza, M. and Catterall, W.A. (2012) Voltage-Gated $\mathrm{Na}^{+}$Channels. In: Noebels, J., Avoli, M., Rogawski, M., Olsen, R. and Delgado-Escueta, A., Eds., Jasper's Basic Mechanisms of the Epilepsies, 4th Edition, Oxford University Press, Oxford, 239-389. https://doi.org/10.1093/med/9780199746545.003.0004

[10] Cheah, C.S., Westenbroek, R.E., Roden, W.H., Kalume, F., Oakley, J.C., Jansen, L.A., et al. (2013) Correlations in Timing of Sodium Channel Expression, Epilepsy, and Sudden Death in Dravet Syndrome. Channels, 7, 468-472.

https://doi.org/10.4161/chan.26023

[11] Duflocq, A., Chareyre, F., Giovannini, M., Couraud, F. and Davenne, M. (2011) Characterization of the Axon Initial Segment (AIS) of Motor Neurons and Identification of a Para-AIS and a Juxtapara-AIS, Organized by Protein 4.1B. BMC Biology, 9, Article No. 66. https://doi.org/10.1186/1741-7007-9-66

[12] Escayg, A., MacDonald, B.T., Meisler, M.H., Baulac, S., Huberfeld, G., An-Gourfinkel, I., Brice, A., LeGuern, E., Moulard, B., Chaigne, D., Buresi, C. and Malafosse, A. (2000) Mutations of SCN1A, Encoding a Neuronal Sodium Channel, in Two Families with GEFS+2. Nature Genetics, 24, 343-345. https://doi.org/10.1038/74159

[13] Oliva, M., Berkovic, S.F. and Petrou, S. (2012) Sodium Channels and the Neurobiology of Epilepsy. Epilepsia, 53, 1849-1859. https://doi.org/10.1111/j.1528-1167.2012.03631.x

[14] Fiest, K.M., Sauro, K.M., Wiebe, S., Patten, S.B., Kwon, C.-S., Dykeman, J., et al. (2017) Prevalence and Incidence of Epilepsy: A Systematic Review and Meta-Analysis 
of International Studies. Neurology, 88, 296-303. https://doi.org/10.1212/WNL.0000000000003509

[15] Banerjee, P.N., Filippi, D. and Hauser, W.A. (2009) The Descriptive Epidemiology of Epilepsy-A Review. Epilepsy research, 85, 31-45. https://doi.org/10.1016/j.eplepsyres.2009.03.003

[16] Marini, C., King, M., Archer, J.S., Newton, M.R. and Berkovic, S.F. (2003) Idiopathic Generalised Epilepsy of Adult Onset: Clinical Syndromes and Genetics. Journal of Neurology, Neurosurgery \& Psychiatry, 74, 192-196. https://doi.org/10.1136/jnnp.74.2.192

[17] Asadi-Pooya, A.A., Emami, M. and Sperling, M.R. (2012) Age of Onset in Idiopathic (Genetic) Generalized Epilepsies: Clinical and EEG Findings in Various Age Groups. Seizure, 21, 417-421.

[18] Taubøll, E., Sveberg, L. and Svalheim, S. (2015) Interactions between Hormones and Epilepsy. Seizure, 28, 3-11.

[19] Ottman, R., Barker-Cummings, C. Leibson, C., Vasoli, V., Hauser, W. and Buchhalter, J. (2011) Accuracy of Family History Information on Epilepsy and Other Seizure Disorders. Neurology, 76, 390-396. https://doi.org/10.1212/WNL.0b013e3182088286

[20] Babtain, F.A. (2013) Impact of a Family History of Epilepsy on the Diagnosis of Epilepsy in Southern Saudi Arabia. Seizure, 22, 542-547.

[21] Oladipo, O., Lesi, F. and Ezeaka, V. (2007) Plasma Magnesium and Calcium Levels in Children with Epilepsy in Lagos. The Nigerian Postgraduate Medical Journal, 14, 26-29.

[22] Bhat, M., Guru, S., Mir, R., Waza, A., Zuberi, M., Sumi, M., et al. (2018) Role of SCN1A and SCN2A Gene Polymorphisms in Epilepsy Syndromes: A Study from India. Journal of Neurology and Neuroscience, 9, Article No. 238.

[23] Nabbout, R., Prud'homme, J., Herman, A., Feingold, J., Brice, A., Dulac, O., et al. (2002) A Locus for Simple Pure Febrile Seizures Maps to Chromosome 6Q22-Q24. Brain, 125, 2668-2680. https://doi.org/10.1093/brain/awf281

[24] Krauss, G. and Barañano, K. (2014) "Idiopathic" and "Genetic" Generalized Epilepsies Intersect: "Idiopathic" and "Genetic" Generalized Epilepsies Intersect. Epilepsy currents, 14, 81-83. https://doi.org/10.5698\%2F1535-7597-14.2.81

[25] Kobow, K., El-Osta, A. and Blümcke, I. (2013) The Methylation Hypothesis of Pharmacoresistance in Epilepsy. Epilepsia, 54, 41-47.

https://doi.org/10.1111/epi.12183

[26] Mirza, N., Appleton, R., Burn, S., Carr, D., Crooks, D., du Plessis, D., et al. (2015) Identifying the Biological Pathways Underlying Human Focal Epilepsy: From Complexity to Coherence to Centrality. Human Molecular Genetics, 24, 4306-4316. https://doi.org/10.1093/hmg/ddv163

[27] Dhiman, V. (2017) Molecular Genetics of Epilepsy: A Clinician's Perspective. Annals of Indian Academy of Neurology, 20, 96-102.

[28] Noebels, J. (2015) Pathway-Driven Discovery of Epilepsy Genes. Nature Neuroscience, 18, 344-350. https://doi.org/10.1038/nn.3933

[29] Crabtree, G.W., Park, A.J., Gordon, J.A. and Gogos, J.A. (2016) Cytosolic Accumulation of L-Proline Disrupts GABA-Ergic Transmission through GAD Blockade. Cell Reports, 17, 570-582. https://doi.org/10.1016/j.celrep.2016.09.029

[30] Treiman, D. (2001) GABAergic Mechanisms in Epilepsy. Epilepsia, 42, 8-12. https://doi.org/10.1046/j.1528-1157.2001.042suppl.3008.x

[31] Anna, A. and Monika, G. (2018) Splicing Mutations in Human Genetic Disorders: 
Examples, Detection, and Confirmation. Journal of Applied Genetics, 59, 253-268. https://doi.org/10.1007/s13353-018-0444-7

[32] Krnjević, K. (1974) Chemical Nature of Synaptic Transmission in Vertebrates. Physiological Reviews, 54, 418-540.

[33] Janjua, N.A., Kabuto, H. and Mori, A. (1992) Increased Plasma Glutamic Acid in a Genetic Model of Epilepsy. Neurochemical Research, 17, 293-296.

https://doi.org/10.1007/BF00966673

[34] Frezza, C. and Mauro, C. (2015) The Metabolic Challenges of Immune Cells in Health and Disease. Frontiers in Immunology, 6, Article No. 293. https://doi.org/10.3389/fimmu.2015.00293

[35] Phaniendra, A., Jestadi, D.B. and Periyasamy, L. (2015) Free Radicals: Properties, Sources, Targets, and Their Implication in Various Diseases. Indian Journal of Clinical Biochemistry, 30, 11-26. https://doi.org/10.1007/s12291-014-0446-0

[36] Keshet, R. and Erez, A. (2018) Arginine and the Metabolic Regulation of Nitric oxide Synthesis in Cancer. Disease Models \& Mechanisms, 11, dmm033332. https://doi.org/10.1242/dmm.033332

[37] Pacher, P., Beckman, J.S. and Liaudet, L. (2007) Nitric Oxide and Peroxynitrite in Health and Disease. Physiological Reviews, 87, 315-424.

[38] Shepherd, G.M. (2004) The Synaptic Organization of the Brain. Oxford University press, Oxford.

[39] Farooqui, T. and Farooqui, A.A. (2011) Lipid-Mediated Oxidative Stress and Inflammation in the Pathogenesis of Parkinson's Disease. Parkinson's Disease, 2011, Article ID: 247467. https://doi.org/10.4061/2011/247467

[40] Rigau, V., Morin, M., Rousset, M.-C., de Bock, F., Lebrun, A., Coubes, P., et al. (2007) Angiogenesis Is Associated with Blood-Brain Barrier Permeability in Temporal Lobe Epilepsy. Brain, 130, 1942-1956. https://doi.org/10.1093/brain/awm118

[41] Stocker, R. and Keaney, J.F. (2004) Role of Oxidative Modifications in Atherosclerosis. Physiological Reviews, 84, 1381-1478. 